PROCEEDINGS OF THE

AMERICAN MATHEMATICAL SOCIETY

Volume 135, Number 1, January 2007, Pages 87-97

S 0002-9939(06)08240-2

Article electronically published on June 13, 2006

\title{
DOUBLE LOGARITHMIC INEQUALITY WITH A SHARP CONSTANT
}

\author{
S. IBRAHIM, M. MAJDOUB, AND N. MASMOUDI \\ (Communicated by Christopher D. Sogge)
}

\begin{abstract}
We prove a $\log \log$ inequality with a sharp constant. We also show that the constant in the Log estimate is "almost" sharp. These estimates are applied to prove a Moser-Trudinger type inequality for solutions of a 2D wave equation.
\end{abstract}

\section{INTRODUCTION AND STATEMENT OF THE RESULTS}

By the Sobolev embedding theorem, it is well known that the Sobolev space $H^{1}\left(\mathbb{R}^{2}\right)$ is embedded in all Lebesgue spaces $L^{p}\left(\mathbb{R}^{2}\right)$ for $2 \leq p<\infty$ but not in $L^{\infty}\left(\mathbb{R}^{2}\right)$. Moreover, $H^{1}$ functions are in a so-called Orlicz space, i.e. their exponential powers are integrable functions. Precisely, we have the following MoserTrudinger inequality (see [1, 11, 14, 16]).

Proposition 1.1. Let $\alpha \in(0,4 \pi)$. A constant $c_{\alpha}$ exists such that

$$
\int_{\mathbb{R}^{2}}\left(\exp \left(\alpha u(x)^{2}\right)-1\right) d x \leq c_{\alpha}\|u\|_{L^{2}}^{2}
$$

for all $u$ in $H^{1}\left(\mathbb{R}^{2}\right)$ such that $\|\nabla u\|_{L^{2}\left(\mathbb{R}^{2}\right)} \leq 1$. Moreover, if $\alpha \geq 4 \pi$, then (11) is false.

Remark 1.2. We point out that $\alpha=4 \pi$ becomes admissible in (1) if we require $\|u\|_{H^{1}\left(\mathbb{R}^{2}\right)} \leq 1$ rather than $\|\nabla u\|_{L^{2}\left(\mathbb{R}^{2}\right)} \leq 1$. Precisely, we have

$$
\sup _{\|u\|_{H^{1}\left(\mathbb{R}^{2}\right)} \leq 1} \int_{\mathbb{R}^{2}}\left(\exp \left(4 \pi u(x)^{2}\right)-1\right) d x<\infty,
$$

and this is false for $\alpha>4 \pi$.

In this paper, we show that we can control the $L^{\infty}$ norm by the $H^{1}$ norm and a stronger norm with a logarithmic growth or double logarithmic growth. The inequality is sharp for the double logarithmic growth.

Recall that $H^{1}$ is the usual Sobolev space endowed with the norm $\|u\|_{H^{1}}^{2}=$ $\|\nabla u\|_{L^{2}}^{2}+\|u\|_{L^{2}}^{2}$. For any real number $\left.\alpha \in\right] 0,1\left[\right.$, we denote by $\dot{\mathcal{C}}^{\alpha}$ the sub-space of $\alpha$ - Hölder continuous functions endowed with the semi-norm

$$
\|u\|_{\dot{\mathcal{C}}^{\alpha}}:=\sup _{x \neq y} \frac{|u(x)-u(y)|}{|x-y|^{\alpha}} .
$$

Received by the editors January 9, 2005 and, in revised form, July 13, 2005.

2000 Mathematics Subject Classification. Primary 49K20, 35L70.

(C)2006 American Mathematical Society 
Also, we denote $\|u\|_{\mathcal{C}^{\alpha}}:=\|u\|_{\dot{\mathcal{C}}^{\alpha}}+\|u\|_{L^{\infty}}$ and define $N_{\alpha}(u)$ to be the ratio $N_{\alpha}(u):=$ $\frac{\|u\|_{\mathcal{C}^{\alpha} \alpha}}{\|\nabla u\|_{L^{2}}}$. For any bounded domain $\Omega$ in $\mathbb{R}^{2}$, define $H_{0}^{1}(\Omega)$ to be the completion in the Sobolev space $H^{1}(\Omega)$ of smooth and compactly supported functions.

The main result of this paper is the following theorem.

Theorem 1.3 (Double logarithmic inequality). Let $\alpha \in] 0,1\left[\right.$ and let $B_{1}$ be the unit ball in $\mathbb{R}^{2}$. Any function in $H_{0}^{1}\left(B_{1}\right) \cap \dot{\mathcal{C}}^{\alpha}\left(B_{1}\right)$ is bounded. Moreover, a positive constant $C_{0}$ exists such that for any function $u \in H_{0}^{1}\left(B_{1}\right) \cap \dot{\mathcal{C}}^{\alpha}\left(B_{1}\right)$, we have

$$
\|u\|_{L^{\infty}}^{2} \leq \frac{1}{2 \pi \alpha}\|\nabla u\|_{L^{2}}^{2} \log \left[e^{3}+C_{0} N_{\alpha}(u) \sqrt{\log \left(2 e+N_{\alpha}(u)\right)}\right],
$$

and the constant $\frac{1}{2 \pi \alpha}$ in (3) is sharp.

Note that $\log (e)=1$. Our second result concerns the following logarithmic inequality.

Theorem 1.4 (Logarithmic inequality). Let $\alpha$ be in $] 0,1[$. For any real number $\lambda>\frac{1}{2 \pi \alpha}$, a constant $C_{\lambda}$ exists such that, for any function $u \in H_{0}^{1}\left(B_{1}\right) \cap \dot{\mathcal{C}}^{\alpha}\left(B_{1}\right)$, we have

$$
\|u\|_{L^{\infty}}^{2} \leq \lambda\|\nabla u\|_{L^{2}}^{2} \log \left(C_{\lambda}+N_{\alpha}(u)\right) .
$$

Moreover, the above inequality does not hold for $\lambda=\frac{1}{2 \pi \alpha}$.

\section{A Littlewood-Paley proof}

To prove the fundamental theorems, we start by showing that inequality (4) can easily be obtained with an unknown absolute constant instead of $\frac{1}{2 \pi \alpha}$. To do so, we give a brief review of the Littlewood-Paley theory, and we refer the reader to [5] for a thorough treatment. Denote by $\mathcal{C}_{0}$ the annular ring defined by

$$
\mathcal{C}_{0}=\left\{\xi \in \mathbb{R}^{2} \text { such that } \frac{3}{4}<|\xi|<\frac{8}{3}\right\},
$$

and choose two nonnegative radial functions $\chi$ and $\varphi$ belonging respectively to $\mathcal{D}(B(0,4 / 3))$ and $\mathcal{D}\left(\mathcal{C}_{0}\right)$ such that

$$
\begin{gathered}
\forall \xi \in \mathbb{R}^{2}, \quad \chi(\xi)+\sum_{j \in \mathbb{N}} \varphi\left(2^{-j} \xi\right)=1, \\
\forall \xi \in \mathbb{R}^{2} \backslash\{0\}, \quad \sum_{j \in \mathbb{Z}} \varphi\left(2^{-j} \xi\right)=1 .
\end{gathered}
$$

Denote $h=\mathcal{F}^{-1} \varphi$ and define the frequency projectors $\Delta_{j}$ and $\dot{\Delta}_{j}$ by

$$
\begin{aligned}
\text { for } j \in \mathbb{Z}, \quad \dot{\Delta}_{j} u & =\varphi\left(2^{-j} D\right) u=2^{2 j} \int_{\mathbb{R}^{2}} h\left(2^{j} y\right) u(x-y) d y, \\
\text { if } j \geq 0, \quad \Delta_{j} u & =\dot{\Delta}_{j} u \\
\Delta_{-1} u & =\chi(D) u=\mathcal{F}^{-1}(\chi(\xi) \hat{u}(\xi)), \\
\text { if } j \leq-2, \quad \Delta_{j} u & =0 .
\end{aligned}
$$

Recall that

$$
\|\nabla u\|_{L^{2}} \sim\left(\sum_{j \in \mathbb{Z}} 2^{2 j}\left\|\dot{\Delta}_{j} u\right\|_{L^{2}}^{2}\right)^{1 / 2}
$$


and

$$
\|u\|_{\dot{\mathcal{C}}^{\alpha}} \sim \sup _{j \in \mathbb{Z}}\left(2^{j \alpha}\left\|\dot{\Delta}_{j} u\right\|_{L^{\infty}}\right)
$$

We mention that $C$ will be used to denote a constant which may vary from line to line.

We have the following result in the whole space.

Proposition 2.1. Let $\alpha$ be in $] 0,1\left[\right.$. For any function $u \in \mathcal{C}^{\alpha}\left(\mathbb{R}^{2}\right) \cap H^{1}\left(\mathbb{R}^{2}\right)$, one has

$$
\|u\|_{L^{\infty}\left(\mathbb{R}^{2}\right)}^{2} \leq C\|u\|_{L^{2}\left(\mathbb{R}^{2}\right)}^{2}+C\|\nabla u\|_{L^{2}\left(\mathbb{R}^{2}\right)}^{2} \log \left(e+N_{\alpha}(u)\right) .
$$

Proof. Write

$$
u=\Delta_{-1} u+\sum_{j=0}^{\infty} \Delta_{j} u=\Delta_{-1} u+\sum_{j=0}^{l-1} \Delta_{j} u+\sum_{j=l}^{\infty} \Delta_{j} u
$$

where $l$ is a nonnegative integer which will be chosen later.

Using Bernstein's inequality, we get

$$
\begin{aligned}
\|u\|_{L^{\infty}} & \leq C\left\|\Delta_{-1} u\right\|_{L^{2}}+C \sum_{j=0}^{l-1} 2^{j}\left\|\Delta_{j} u\right\|_{L^{2}}+\sum_{j=l}^{\infty} 2^{-j \alpha}\left(2^{j \alpha}\left\|\Delta_{j} u\right\|_{L^{\infty}}\right) \\
& \leq C\|u\|_{L^{2}}+C \sqrt{l}\left(\sum_{j=0}^{l-1} 2^{2 j}\left\|\Delta_{j} u\right\|_{L^{2}}^{2}\right)^{1 / 2}+C\left(\sum_{j=l}^{\infty} 2^{-j \alpha}\right)\|u\|_{\dot{\mathcal{C}}^{\alpha}} \\
& \leq C\left(\|u\|_{L^{2}}+\sqrt{l}\|\nabla u\|_{L^{2}}+\frac{2^{-\alpha l}}{1-2^{-\alpha}}\|u\|_{\dot{\mathcal{C}}^{\alpha}}\right)
\end{aligned}
$$

so

$$
\|u\|_{L^{\infty}}^{2} \leq C\left(\|u\|_{L^{2}}^{2}+l\|\nabla u\|_{L^{2}}^{2}+\frac{2^{-2 \alpha l}}{\left(1-2^{-\alpha}\right)^{2}}\|u\|_{\dot{\mathcal{C}}^{\alpha}}^{2}\right) .
$$

Denoting by $] x[$ the integer part of the real number $x$ and choosing

$$
l:=\operatorname{Max}(1,1+] 2 \log _{2}\left(N_{\alpha}(u)^{2}\right)[),
$$

the proof of Proposition 2.1 is achieved.

Clearly, if $u$ is supported in the unit ball $B_{1}$, then using the Poincaré inequality and Proposition 2.1, we get

$$
\|u\|_{L^{\infty}}^{2} \leq C\|\nabla u\|_{L^{2}}^{2} \log \left(C_{0}+N_{\alpha}(u)\right),
$$

for some constant $C_{0}$ big enough.

\section{Proof of Theorem 1.3}

To prove (3) and the fact that the constant is sharp, it is sufficient to show that

$$
2 \pi \alpha=\inf _{u \in H_{0}^{1}\left(B_{1}\right) \cap \dot{\mathcal{C}}^{\alpha}\left(B_{1}\right)} \frac{\|\nabla u\|_{L^{2}}^{2} \log \left[e^{3}+C_{0} N_{\alpha}(u) \sqrt{\log \left(2 e+N_{\alpha}(u)\right)}\right]}{\|u\|_{L^{\infty}}^{2}},
$$


for any $C_{0}$ big enough. Let us start by proving the sharpness of the constant. Define $u_{k}(x)=f_{k}(-2 \log |x|)$, where for all nonnegative integer $k$

$$
f_{k}(t)=\left\{\begin{array}{cll}
0 & \text { if } & t \leq 0, \\
\sqrt{\frac{k}{4 \pi}} \frac{t}{k} & \text { if } & 0 \leq t \leq k, \\
\sqrt{\frac{k}{4 \pi}} & \text { if } & t \geq k .
\end{array}\right.
$$

These functions were introduced in [1] to show the optimality of the exponent $4 \pi$ in Trudinger-Moser inequality (see also [1] and 10]). An easy computation shows that $\left\|\nabla u_{k}\right\|_{L^{2}}^{2}=1$. By interpolation and since $f_{k}$ is nonnegative, we have

$$
\left\|u_{k}\right\|_{\dot{\mathcal{C}}^{\alpha}} \leq\left\|u_{k}\right\|_{L^{\infty}}^{1-\alpha}\left\|u_{k}\right\|_{L i p}^{\alpha}
$$

where $\left\|u_{k}\right\|_{L i p}=\sup _{x \neq y} \frac{\left|u_{k}(x)-u_{k}(y)\right|}{|x-y|}$. Hence

$$
\left\|u_{k}\right\|_{\dot{\mathcal{C}}^{\alpha}} \leq C k^{\frac{1}{2}-\alpha} \exp \left(\frac{\alpha k}{2}\right)
$$

Denoting by

$$
R(u):=\frac{\|\nabla u\|_{L^{2}}^{2} \log \left[e^{3}+C_{0} N_{\alpha}(u) \sqrt{\log \left(2 e+N_{\alpha}(u)\right)}\right]}{\|u\|_{L^{\infty}}^{2}},
$$

it is clear that

$$
R\left(u_{k}\right) \geq \inf _{u \in H_{0}^{1}\left(B_{1}\right) \cap \dot{\mathcal{C}}^{\alpha}\left(B_{1}\right)} \frac{\|\nabla u\|_{L^{2}}^{2} \log \left[e^{3}+C_{0} N_{\alpha}(u) \sqrt{\log \left(2 e+N_{\alpha}(u)\right)}\right]}{\|u\|_{L^{\infty}}^{2}} .
$$

Taking the limit as $k \rightarrow \infty$, we deduce that

$$
2 \pi \alpha \geq \inf _{u \in H_{0}^{1}\left(B_{1}\right) \cap \dot{\mathcal{C}}^{\alpha}\left(B_{1}\right)} \frac{\|\nabla u\|_{L^{2}}^{2} \log \left[e^{3}+C_{0} N_{\alpha}(u) \sqrt{\log \left(2 e+N_{\alpha}(u)\right)}\right]}{\|u\|_{L^{\infty}}^{2}} .
$$

To prove (3), we start by noting that for any function $u$, the norms $\|\nabla u\|_{L^{2}}$ and $\|u\|_{\mathcal{C}^{\alpha}}$ are nonincreasing under symmetric nonincreasing rearrangements, while $\|u\|_{L^{\infty}}$ remains unchanged.

Using the fact that for all $C>0$

$$
t \rightarrow f(t):=t^{2} \log \left[e^{3}+\frac{C}{t} \sqrt{\left[\log \left(2 e+\frac{1}{t}\right)\right]}\right]
$$

is increasing, it is sufficient to check the minimizer figured in (17) in the class of nonnegative, nonincreasing and radially symmetric functions.

Without loss of generality, we can normalize $\|u\|_{L^{\infty}}$ to be equal to 1 . Since $u$ vanishes on the boundary, we deduce that $\|u\|_{\dot{\mathcal{C}}^{\alpha}}$ is larger than or equal to 1 . Moreover, if $\|u\|_{\dot{\mathcal{C}}^{\alpha}}=1$, then necessarily, $u(x)=1-|x|^{\alpha}$ and the inequality is trivial. In the sequel, we will assume that $\|u\|_{\mathcal{C}^{\alpha}}>1$.

Let $H_{0, \text { rad }}^{1}\left(B_{1}\right)$ be the space of all nonincreasing and radially symmetric functions in $H_{0}^{1}\left(B_{1}\right)$. For any parameter $D>1$, we denote by $K_{D}$ the closed convex subset of $H_{0, \text { rad }}^{1}\left(B_{1}\right)$ defined by

$$
\left.\left.K_{D}=\left\{u \in H_{0, \text { rad }}^{1}\left(B_{1}\right): u(r) \geq 1-D r^{\alpha}, \quad r \in\right] 0,1\right]\right\} .
$$


Note that the set of radially symmetric functions which satisfy $\|u\|_{\dot{\mathcal{C}}^{\alpha}} \leq D$ is included in $K_{D}$. Hence, to get the result, it is sufficient to prove that

$$
2 \pi \alpha \leq \inf _{D \geq 1} \inf _{\left\{u \in K_{D},\|u\|_{\left.L^{\infty}=1,\|u\|_{\mathcal{C}^{\alpha}}=D\right\}}\right.}\|\nabla u\|_{L^{2}}^{2} \log \left[e^{3}+\frac{C_{0} D}{\|\nabla u\|_{L^{2}}} \sqrt{\log \left(2 e+\frac{D}{\|\nabla u\|_{L^{2}}}\right)}\right]
$$

or just that

$$
2 \pi \alpha \leq \inf _{D \geq 1} \inf _{\left\{u \in K_{D}\right\}}\|\nabla u\|_{L^{2}}^{2} \log \left[e^{3}+\frac{C_{0} D}{\|\nabla u\|_{L^{2}}} \sqrt{\log \left(2 e+\frac{D}{\|\nabla u\|_{L^{2}}}\right)}\right] .
$$

Consider the problem of minimizing

$$
I[u]:=\|\nabla u\|_{L^{2}\left(B_{1}\right)}^{2}
$$

among all the functions belonging to the set $K_{D}$. This is a variational problem with obstacle. It is well known (see, for example, Kinderlehrer-Stampacchia 9] and L. C. Evans [6]) that it has a unique minimizer $u^{*}$ which is variationally characterized by

$$
\int_{B_{1}} \nabla u^{*} \cdot \nabla v d x \geq\left\|\nabla u^{*}\right\|_{L^{2}\left(B_{1}\right)}^{2},
$$

for any $v \in K_{D}$. Moreover $u^{*}$ is in the Sobolev space $W^{2, \infty}\left(B_{1}\right)$. Hence the radially symmetric set

$$
\mathcal{O}:=\left\{x \in B_{1}: u^{*}(x)>1-D|x|^{\alpha}\right\}
$$

is open and $u^{*}$ is harmonic in $\mathcal{O}$. On the other hand, note that any radially symmetric harmonic functions in $\mathbb{R}^{2}$ can only coincide in a unique tangent point with the function $r \rightarrow 1-D r^{\alpha}$. Note also that because of the boundary condition at $r=1, u^{*}$ cannot start to be harmonic near $r=0$. Therefore there exists a unique $a \in] 0,1[$ such that

$$
\begin{aligned}
& u^{*}(r)=1-D r^{\alpha} \text { if } r \in[0, a], \\
& u^{*}(r)=\left(1-D a^{\alpha}\right) \frac{\log r}{\log a} \text { if } r \in[a, 1],
\end{aligned}
$$

also satisfy the tangent condition

$$
a^{\alpha}=\frac{1-D a^{\alpha}}{D\left|\log \left(a^{\alpha}\right)\right|} .
$$

Note that if $D \rightarrow 1$, then $a \rightarrow 1$, and therefore (12) still makes sense in the limit case.

In particular, note that $\left\|u^{*}\right\|_{L^{\infty}}=1,\left\|u^{*}\right\|_{\dot{\mathcal{C}}^{\alpha}}=D$, and

$$
\left\|\nabla u^{*}\right\|_{L^{2}}^{2}=\pi \alpha D^{2} a^{2 \alpha}-2 \pi\left(\frac{1-D a^{\alpha}}{\log (a)}\right)^{2} \log (a) .
$$

Substituting $D$ from (12) into (13), we get

$$
\left\|\nabla u^{*}\right\|_{L^{2}}^{2}=2 \pi \alpha \frac{1 / 2-\log \left(a^{\alpha}\right)}{\left(1-\log \left(a^{\alpha}\right)\right)^{2}} .
$$

Denoting by $\left.x:=a^{\alpha} \in\right] 0,1[$, we have

$$
\left\|\nabla u^{*}\right\|_{L^{2}}^{2}=2 \pi \alpha \frac{1 / 2-\log (x)}{(1-\log (x))^{2}}
$$


and

$$
\left\|u^{*}\right\|_{\dot{\mathcal{C}}^{\alpha}}=\frac{1}{x(1-\log (x))}
$$

Setting

and

$$
g(x):=\frac{1}{x \sqrt{2 \pi \alpha(1 / 2-\log (x))}}
$$

$$
F_{C}(x):=\frac{\frac{1}{2}-\log (x)}{(1-\log (x))^{2}} \log \left[e^{3}+C g(x) \sqrt{\log (2 e+g(x))}\right],
$$

it is sufficient to show that a constant $C_{0}$ exists such that for all $0<x \leq 1$, the function $F_{C_{0}}$ satisfies

$$
F_{C_{0}}(x) \geq 1 .
$$

First, observe that for every $0<x \leq 1$

$$
\frac{\frac{1}{2}-\log (x)}{(1-\log (x))^{2}} \geq \frac{1}{(2-\log (x))} .
$$

Hence for any $C>0$, (16) holds if $2-\log x \leq 3$, namely if $x \geq 1 / e$.

In the sequel, we suppose that $x \leq 1 / e$, hence

$$
\begin{aligned}
F(x) \geq & \frac{1}{(2-\log (x))}\left[-\log (x)+\log \left(\frac{C_{0}}{\sqrt{2 \pi \alpha}}\right)-\frac{1}{2} \log (1 / 2-\log (x))\right. \\
& \left.\quad+\frac{1}{2} \log (\log (2 e+g(x)))\right] \\
\geq & 1+\frac{1}{(2-\log (x))}\left[\log \left(\frac{C_{0}}{e^{2} \sqrt{2 \pi \alpha}}\right)+\frac{1}{2} \log \left(\frac{\log (2 e+g(x))}{(1 / 2-\log (x))}\right)\right] .
\end{aligned}
$$

The function $h(x)=\frac{\log (2 e+g(x))}{(1 / 2-\log (x))}$ is bounded away from zero on $(0,1 / e)$. Hence, we can find $C_{0}$ big enough such that the second term on the right-hand side of (17) is non-negative. This achieves the proof of Theorem 1.3

\section{Proof of Theorem 1.4}

The proof of Theorem 1.4 is similar to that of Theorem 1.3. Indeed, consider $u^{*}$ the minimizer of the Dirichlet norm (9) among all functions in $K_{D}$ defined in (8). Note that according to (14) and (15), we have

$$
\left\|\nabla u^{*}\right\|_{L^{2}}^{2} \log \left(C_{\lambda}+N_{\alpha}\left(u^{*}\right)\right):=H(x),
$$

where

$$
H(x)=2 \pi \alpha \frac{1 / 2-\log (x)}{(1-\log (x))^{2}} \log \left(C_{\lambda}+\frac{1}{x \sqrt{2 \pi \alpha(1 / 2-\log (x))}}\right) .
$$

Taking $C_{\lambda}=e$ in $H(x)$, we see that $H(x)$ goes to $2 \pi \alpha$ as $x$ goes to 0 . Hence, for any $\lambda>\frac{1}{2 \pi \alpha}$, there exists $x_{\lambda}>0$ such that $\lambda H(x) \geq 1$, for any $0<x<x_{\lambda}$ and $C_{\lambda} \geq e$. Now, if $x \in\left[x_{\lambda}, 1\right]$, choosing the constant $C_{\lambda}>e$ big enough such that

$$
\frac{1 / 2}{\left(1-\log \left(x_{\lambda}\right)\right)^{2}} \log \left(C_{\lambda}\right) \geq 1
$$

we see that $\lambda H(x) \geq 1$. Hence, by this choice of $C_{\lambda}$, we see that $\lambda H(x) \geq 1$ for all $0<x \leq 1$. This achieves the proof of (4). 
Now, let us prove that (4) does not hold for $\lambda=\frac{1}{2 \pi \alpha}$. More precisely, we will prove that a sequence of functions $\left(u_{n}\right)_{n}$ exists such that $u_{n} \in H_{0}^{1}\left(B_{1}\right) \cap \dot{\mathcal{C}}^{\alpha}\left(B_{1}\right)$ and for $n$ big enough the following holds:

$$
\left\|u_{n}\right\|_{L^{\infty}}^{2}>\frac{1}{2 \pi \alpha}\left\|\nabla u_{n}\right\|_{L^{2}}^{2} \log \left(n^{1 / 4}+n^{1 / 4} N_{\alpha}\left(u_{n}\right)\right) .
$$

Let $u_{n}$ be the radially symmetric function defined by

$$
u_{n}(r)=1-e^{n} r^{\alpha} \text { if } r \in\left[0, a_{n}\right] \text {, and } u_{n}(r)=\left(1-e^{n} a_{n}^{\alpha}\right) \frac{\log r}{\log a_{n}} \text { if } r \in\left[a_{n}, 1\right],
$$

where $a_{n}$ is chosen such that $a_{n}^{\alpha}:=x_{n}$ is the unique solution in $(0,1)$ of the equation $x=\frac{1-e^{n} x}{e^{n}|\log (x)|}$. Note indeed that the function $h(x)=e^{n}(x+x|\log (x)|)$ is increasing on $(0,1)$. Hence, we see easily that

$$
\frac{e^{-n}}{n \log (n)} \leq x_{n} \leq \frac{e^{-n}}{n}
$$

Obviously, this construction is inspired from the minimizer of the variational problem with obstacle described in Section 3 where we have chosen $D_{n}=e^{n}$. Hence, according to (14) and (15), we have

$$
\left\|\nabla u_{n}\right\|_{L^{2}}^{2}=2 \pi \alpha \frac{1 / 2-\log \left(x_{n}\right)}{\left(1-\log \left(x_{n}\right)\right)^{2}}
$$

and

$$
\left\|u_{n}\right\|_{\dot{\mathcal{C}}^{\alpha}}=\frac{1}{x_{n}\left(1-\log \left(x_{n}\right)\right)} .
$$

Now to prove (18), it is sufficient to prove that for $n$ big enough we have

$$
h_{n}:=\frac{\frac{1}{2}-\log \left(x_{n}\right)}{\left(1-\log \left(x_{n}\right)\right)^{2}} \log \left[n^{1 / 4}+\frac{n^{1 / 4}}{x_{n} \sqrt{2 \pi \alpha\left(1 / 2-\log \left(x_{n}\right)\right)}}\right]<1 .
$$

Note that using (19), we have

$$
h_{n}<\frac{\frac{1}{2}+n+\log (n)+\log \log n}{(1+\log (n)+n)^{2}} \log \left[n^{1 / 4}+\frac{n^{1 / 4} e^{n} n \log n}{\sqrt{2 \pi \alpha n}}\right] .
$$

Hence $h_{n}<1-\frac{1}{4} \frac{\log n}{n}+o\left(\frac{\log n}{n}\right)$, which is strictly less than 1 if $n$ is sufficiently large. The proof of (18) is achieved.

\section{Case of the whole SPace}

Theorems 1.3 and 1.4 were stated in the ball of radius one. If the function $u$ is supported in a bigger ball $B_{R}=B(0, R)$, then a simple scaling argument shows that

$$
\|u\|_{L^{\infty}\left(B_{R}\right)}^{2} \leq \frac{1}{2 \pi \alpha}\|\nabla u\|_{L^{2}\left(B_{R}\right)}^{2} \log \left[e^{3}+C_{0} R^{\alpha} N_{\alpha}(u) \sqrt{\log \left(2 e+R^{\alpha} N_{\alpha}(u)\right)}\right] .
$$

Remark 5.1. Using symmetric nonincreasing rearrangement of functions, the results of Theorem 1.3 and Theorem 1.4 remain true for any bounded and regular domain $\Omega$ of $\mathbb{R}^{2}$. Precisely, if $f \in H_{0}^{1}(\Omega) \cap \dot{\mathcal{C}}^{\alpha}(\Omega)$, then its corresponding symmetric nonincreasing function, usually denoted by $f^{\star}$, is in $H_{0}^{1}\left(B_{R}\right) \cap \dot{\mathcal{C}}^{\alpha}\left(B_{R}\right)$, where $R=\sqrt{\frac{|\Omega|}{2 \pi}}$. We refer to [15], 2] for the definition, the properties and applications 
of rearrangements of functions. Applying the results of Theorem 1.3 and Theorem 1.4 to $f^{\star}$ and using the fact that

$$
\begin{gathered}
\left\|f^{\star}\right\|_{L^{\infty}}=\|f\|_{L^{\infty}}, \\
\left\|\nabla f^{\star}\right\|_{L^{2}} \leq\|\nabla f\|_{L^{2}}, \quad\left\|f^{\star}\right\|_{\dot{\mathcal{C}}^{\alpha}} \leq\|f\|_{\dot{\mathcal{C}}^{\alpha}},
\end{gathered}
$$

we get the result for a general domain $\Omega$.

Note that this estimate cannot be extended to the whole space since $R^{\alpha}$ diverges. Instead, we have the following result concerning the whole space.

Corollary 5.2. Let $\alpha \in] 0,1\left[\right.$. For any $\lambda>\frac{1}{2 \pi \alpha}$ and any $0<\mu \leq 1$, a constant $C_{\lambda}>0$ exists such that, for any function $u \in H^{1}\left(\mathbb{R}^{2}\right) \cap \mathcal{C}^{\alpha}\left(\mathbb{R}^{2}\right)$

$$
\|u\|_{L^{\infty}}^{2} \leq \lambda\|u\|_{\mu}^{2} \log \left(C_{\lambda}+\frac{8^{\alpha} \mu^{-\alpha}\|u\|_{\mathcal{C}^{\alpha}}}{\|u\|_{\mu}}\right)
$$

where $\|u\|_{\mu}^{2}=\|\nabla u\|_{L^{2}}^{2}+\mu^{2}\|u\|_{L^{2}}^{2}$.

Proof. Let $u$ be a function in $H^{1}\left(\mathbb{R}^{2}\right) \cap \mathcal{C}^{\alpha}\left(\mathbb{R}^{2}\right), \lambda>\frac{1}{2 \pi \alpha}$ and $0<\mu \leq 1$. Fix a radially symmetric function $\varphi$ in $\mathcal{C}_{0}^{\infty}\left(B_{4}\right)$ satisfying $0 \leq \varphi \leq 1, \varphi \equiv 1$ for $r$ near 0 , $\left|\partial_{r} \varphi\right| \leq 1$ and $|\Delta \varphi| \leq 1$. Define $\varphi_{\mu}$ by $\varphi_{\mu}(x)=\varphi\left(\frac{\mu}{2}|x|\right)$.

Without loss of generality, we can assume that $\|u\|_{L^{\infty}}=|u(0)|$. Note that in particular one has

$$
\begin{gathered}
\left\|\varphi_{\mu} u\right\|_{\dot{\mathcal{C}}^{\alpha}} \leq\|u\|_{\mathcal{C}^{\alpha}} \\
\left\|\nabla\left(\varphi_{\mu} u\right)\right\|_{L^{2}}^{2} \leq\|\nabla u\|_{L^{2}}^{2}+\frac{\mu^{2}}{4}\|u\|_{L^{2}}^{2}+2 \int_{\mathbb{R}^{2}} \varphi_{\mu} u \nabla \varphi_{\mu} \nabla u d x
\end{gathered}
$$

Integrating by parts,

$$
2 \int_{\mathbb{R}^{2}} \varphi_{\mu} u \nabla \varphi_{\mu} \nabla u d x=-\frac{1}{2} \int_{R^{2}} \Delta \varphi_{\mu}^{2} u^{2} d x=-\frac{\mu^{2}}{8} \int_{R^{2}} \Delta \varphi^{2}\left(\frac{\mu}{2} x\right) u^{2} d x .
$$

Hence,

$$
\left\|\nabla\left(\varphi_{\mu} u\right)\right\|_{L^{2}}^{2} \leq\|\nabla u\|_{L^{2}}^{2}+\mu^{2}\|u\|_{L^{2}}^{2}
$$

Applying Theorem 1.4 in the ball $B_{8 / \mu}$ and using the fact that for any constant $C>0$ the function $x \rightarrow x^{2} \log \left(C_{\lambda}+\frac{C}{x}\right)$ is increasing, the proof of Corollary 5.2 is achieved.

We also have the following result.

Corollary 5.3. Let $\alpha \in] 0,1\left[\right.$. For any $\lambda>\frac{1}{2 \pi \alpha}$, a constant $C_{\lambda}>0$ exists such that, for any function $u \in H^{1}\left(\mathbb{R}^{2}\right) \cap \mathcal{C}^{\alpha}\left(\mathbb{R}^{2}\right)$,

$$
\|u\|_{L^{\infty}} \leq\|u\|_{L^{2}}+\|\nabla u\|_{L^{2}} \sqrt{\lambda \log \left(e+C_{\lambda} \frac{\|u\|_{\mathcal{C}^{\alpha}}}{\|\nabla u\|_{L^{2}}}\right)} .
$$

For the proof of Corollary 5.3, we take the Littlewood-Paley decomposition of $u$, $u=\Delta_{-1} u+v$, where $v=\sum_{j=0}^{\infty} \Delta_{j} u$. Hence $\|v\|_{L^{2}} \leq C\|\nabla v\|_{L^{2}}$ and $\|v\|_{\mathcal{C}^{\alpha}} \leq\|u\|_{\mathcal{C}^{\alpha}}$. So

$$
\|u\|_{L^{\infty}} \leq\left\|\Delta_{-1} u\right\|_{L^{\infty}}+\|v\|_{L^{\infty}} .
$$

Then, we apply Corollary 5.2 to $v$ with $\lambda^{\prime}$ and $\mu^{\prime}$ such that $\lambda^{\prime}\left(1+C^{2} \mu^{\prime 2}\right)<\lambda$.

Of course, we have similar inequalities for the $\log \log$ inequality (3) in $\mathbb{R}^{2}$ with the sharp constant $\frac{1}{2 \pi \alpha}$. 


\section{Application to the Wave equation}

Corollary 5.2 is useful in the study of the Cauchy problem associated with the following type of 2D-nonlinear wave equation

$$
\partial_{t}^{2} u-\Delta u+u+u\left(\exp \left(4 \pi u^{2}\right)-1\right)=0
$$

with initial data $u(0, \cdot)=f, \partial_{t} u(0, \cdot)=g$, where $(f, g) \in H^{1}\left(\mathbb{R}^{2}\right) \times L^{2}\left(\mathbb{R}^{2}\right)$ (see [8] for more details). For such a problem, only global (in time) wellposedness for small data or local wellposedness for radially symmetric data $(0, g)$ satisfying $\|g\|_{L^{2}} \leq 1$ are known so far. See [12, [13] and [3. To establish an energy estimate for solutions of (22), we need to estimate the source term $u\left(\exp \left(4 \pi u^{2}\right)-1\right)$ in $L_{t}^{1}\left(L_{x}^{2}\right)$ (or any other dual Strichartz norm). The problem with taking the $L_{x}^{2}$ norm is that the factor $4 \pi$ appearing in the exponential will be doubled, and hence, we cannot apply the Moser-Trudinger inequality if $\|u\|_{H^{1}}>\frac{1}{\sqrt{2}}$.

In the following, we show how Corollary 5.2 enables us to overcome this difficulty and allows us to deal with solutions such that $\|u\|_{H^{1}} \leq 1$. This seems to be optimal [8]. For simplicity, we assume that $u$ solves the "linearized problem"; this corresponds to the first iteration in a proof based on the Picard scheme.

In the sequel, we assume that $(f, g) \in H^{1}\left(\mathbb{R}^{2}\right) \times L^{2}\left(\mathbb{R}^{2}\right)$ such that

$$
\|f\|_{H^{1}}^{2}+\|g\|_{L^{2}}^{2} \leq 1 \text {. }
$$

Denote by $v$ the solution of the 2D linear Klein-Gordon equation

$$
\begin{aligned}
\partial_{t}^{2} v-\Delta v+v & =0, \\
v(0, \cdot)=f, \quad \partial_{t} v(0, \cdot) & =g .
\end{aligned}
$$

Since the energy $\|\nabla v(t, \cdot)\|_{L^{2}\left(\mathbb{R}^{2}\right)}^{2}+\|v(t, \cdot)\|_{L^{2}\left(\mathbb{R}^{2}\right)}^{2}+\left\|\partial_{t} v(t, \cdot)\right\|_{L^{2}\left(\mathbb{R}^{2}\right)}^{2}$ is conserved, $v(t, \cdot)$ remains in the unit ball of $H^{1}$ uniformly in time. So according to (2) we have

$$
\sup _{t \in \mathbb{R}} \int_{\mathbb{R}^{2}}\left(e^{4 \pi v(t, x)^{2}}-1\right) d x \leq C,
$$

which means that $\exp \left(4 \pi v^{2}\right)-1 \in L^{\infty}\left(\mathbb{R} ; L^{1}\left(\mathbb{R}^{2}\right)\right)$. For any $\mu>0$, denote

$$
E_{\mu}(t):=\|\nabla v(t, \cdot)\|_{L^{2}\left(\mathbb{R}^{2}\right)}^{2}+\mu^{2}\|v(t, \cdot)\|_{L^{2}\left(\mathbb{R}^{2}\right)}^{2} \cdot
$$

The following result will enable us to estimate $\exp \left(4 \pi v^{2}\right)-1$ in $L_{l o c}^{1}\left(\mathbb{R} ; L^{2}\left(\mathbb{R}^{2}\right)\right)$.

Proposition 6.1. Let $v$ be the solution of (24) with initial data satisfying (23). For any $T>0$ and $0<\mu<1$, a nonnegative constant $C$ exists such that

$$
\int_{0}^{T}\left\|\exp \left(4 \pi v^{2}(t, \cdot)\right)-1\right\|_{L^{2}\left(\mathbb{R}^{2}\right)} d t \leq C
$$

Proof. Recall that since $v \in \mathcal{C}\left(\mathbb{R}, H^{1}\right) \cap \mathcal{C}^{1}\left(\mathbb{R}, L^{2}\right)$, the function $t \longrightarrow E_{\mu}(t)$ is continuous. The energy conservation satisfied by $v$ shows that

$$
\left\|\partial_{t} v(t, \cdot)\right\|_{L^{2}\left(\mathbb{R}^{2}\right)}^{2}+E_{1}(t)=E_{1}(0)+\|g\|_{L^{2}}^{2} \leq 1 .
$$

Now, fix $\mu<1$ and $T>0$. There exists a time $\tau=\tau(\mu, T) \leq T$ such that

$$
\sup _{t \in[0, T]} E_{\mu}(t)=E_{\mu}(\tau)<1
$$

For almost every $t$ we have

$$
\int_{\mathbb{R}^{2}}\left(\exp \left(4 \pi v^{2}(t, x)\right)-1\right)^{2} d x \leq\left\|\exp \left(4 \pi v^{2}(t, \cdot)\right)-1\right\|_{L^{1}} \exp \left(4 \pi\|v(t, \cdot)\|_{L^{\infty}}^{2}\right)
$$


Note that, thanks to the conservation of the energy and the Moser-Trudinger inequality, the first factor in the above inequality is uniformly bounded. On the other hand, choosing $\alpha=\frac{1}{4}$ in (20) we obtain, for any $\lambda>\frac{2}{\pi}$,

$$
\exp \left(2 \pi\|v(t, \cdot)\|_{L^{\infty}}^{2}\right) \leq\left(C+\frac{\|v(t, \cdot)\|_{\mathcal{C}^{1 / 4}}}{E_{\mu}(\tau)^{1 / 2}}\right)^{2 \pi \lambda E_{\mu}(\tau)} .
$$

Using the fact that the bound given on the right-hand side of (26) is increasing in $E_{\mu}(\tau)$, we can assume that $E_{\mu}(\tau)>1 / 2$. Since $E_{\mu}(\tau)<1$, one can choose $\lambda>\frac{2}{\pi}$ such that $\beta:=2 \pi \lambda E_{\mu}(\tau)<4$. Hence, we have

$$
\begin{aligned}
\int_{0}^{T} \exp \left(2 \pi\|v(t, \cdot)\|_{L^{\infty}}^{2}\right) d t & \leq C \int_{0}^{T}\left(C+\|v(t, \cdot)\|_{\mathcal{C}^{1 / 4}}\right)^{\beta} d t \\
& \leq C T^{1-\frac{\beta}{4}}\left(\int_{0}^{T}\left(C+\|v(t, \cdot)\|_{\mathcal{C}^{1 / 4}}\right)^{4} d t\right)^{\frac{\beta}{4}} .
\end{aligned}
$$

Now, thanks to the so-called Strichartz estimates (see [4, 7]), we have $v \in$ $L^{4}\left(\mathbb{R}, \mathcal{C}^{1 / 4}\left(\mathbb{R}^{2}\right)\right)$, and therefore Proposition 6.1 is proved.

Remark 6.2. To study the Cauchy problem for (22), we need a bound in $L_{T}^{1}\left(L^{2}\right)$ for $u\left(\exp \left(4 \pi u^{2}\right)-1\right)$. Using Hölder inequality we have

$$
\left\|v\left(\exp \left(4 \pi v^{2}(t, \cdot)\right)-1\right)\right\|_{L^{2}\left(\mathbb{R}^{2}\right)} \leq\left\|\exp \left(4 \pi v^{2}(t, \cdot)\right)-1\right\|_{L^{2(1+\varepsilon)}\left(\mathbb{R}^{2}\right)}\|v(t, \cdot)\|_{L^{2\left(1+\frac{1}{\varepsilon}\right)\left(\mathbb{R}^{2}\right)}} \cdot
$$

Following the same proof as that of the above proposition and suitably choosing $\varepsilon>0$, we can prove a bound for $\left\|\exp \left(4 \pi v^{2}\right)-1\right\|_{L_{T}^{1}\left(L^{2+2 \varepsilon}\right)}$.

\section{ACKNOWLEDGMENT}

The first author would like to thank W. Craig and A. Biryuk for fruitful discussions and the Fields Institute and McMaster University for their support. He was also supported by the NSERC grant 238452-01. The second author was supported by the Laboratoire d' EDP of the Faculty of Sciences of Tunis. The third author was partially supported by an NSF grant and by an Alfred P. Sloan Fellowship.

\section{REFERENCES}

[1] S. Adachi and K. Tanaka: Trudinger type inequalities in $\mathbb{R}^{N}$ and their best exponents, Proc. Amer. Math. Society, 128, N. 7, 2051-2057, 1999. MR1646323 (2000m:46069)

[2] A. Alvino, P.-L. Lions and G. Trombetti: On optimization problem with prescribed rearrangements, Nonlinear Anal. T. M. A. 13, 185-220, 1989. MR0979040 (90c:90236)

[3] A. Atallah Baraket: Local existence and estimations for a semilinear wave equation in two dimension space, Boll. Unione Mat. Ital. Sez. B Artic. Ric. Mat., 8, 1, 1-21, 2004. MR2044259 (2005a:35198)

[4] P. Brenner: On space-time means and everywhere defined scattering operators for nonlinear Klein-Gordon equations, Math. Z., 186, 383-391, 1984. MR0744828(85h:35183)

[5] J.-Y. Chemin: Fluides parfaits incompressibles, Astérisque no. 230, Société Mathématiques de France, 1995. MR1340046 (97d:76007)

[6] L. C. Evans: Partial differential equations, Graduate Studies in Mathematics, AMS, 1998. MR:1625845 (99e:35001)

[7] J. Ginibre and G. Velo: Time decay of finite energy solutions of the nonlinear KleinGordon and Schrodinger equations, Ann. Inst. Poincaré Phys. Théo., 43, 399-442, 1985. MR0824083 (87g:35208)

[8] S. Ibrahim, M. Majdoub and N. Masmoudi: Global solutions for a semilinear $2 D$ Klein-Gordon equation with exponential type nonlinearity, to appear in Communications in Pure and Applied Mathematics. 
[9] D. Kinderlehrer and G. Stampacchia: An introduction to variational inequalities and their applications, Academic Press, 1980. MR.0567696 (81g:49013)

[10] P.-L. Lions: The concentration-compactness principal in the calculus of variations. The limit case, Rev. Mat. Iberoamericana, 1, 12-45, 1985. MR0834360 (87c:49007)

[11] J. Moser: A sharp form of an inequality of N. Trudinger, Ind. Univ. Math. J., 20, 10771092, 1971. MR.0301504 (46:662)

[12] M. Nakamura and T. Ozawa: Global solutions in the critical Sobolev space for the wave equations with nonlinearity of exponential growth, Math. Z., 231, 479-487, 1999. MR.1704989 (2001b:35216)

[13] M. Nakamura and T. Ozawa: The Cauchy problem for nonlinear wave equations in the Sobolev space of critical order, Discrete and Continuous Dynamical Systems, 5, no. 1, 215-231, 1999. MR1664497 (99k:35128)

[14] B. Ruf: A sharp Trudinger-Moser type inequality for unbounded domains in $\mathbb{R}^{2}$, J. Funct. Anal. 219 (2005), 340-367. MR2109256 (2005k:46082)

[15] G. Talenti: Inequalities in rearrangement invariant function spaces, Nonlinear analysis, function spaces and applications, 5(Prague), 177-230, 1994. MR1322313 (96a:46062)

[16] N.S. Trudinger: On imbedding into Orlicz spaces and some applications, J. Math. Mech., 17, 473-484, 1967. MR0216286(35:7121)

Department of Mathematics and Statistics, McMaster University, 1280 Main Street West, Hamilton, Ontario, Canada L8S 4L8

E-mail address: ibrahims@math.mcmaster.ca

Département de Mathématiques, Faculté des Sciences de Tunis, Campus Universitaire 1060, Tunis, Tunisia

E-mail address: mohamed.majdoub@fst.rnu.tn

Department of Mathematics, Courant Institute of Mathematical Sciences, New York University, 251 Mercer St., New York, New York 10012

E-mail address: masmoudi@cims.nyu.edu 